\title{
Analysis of direct-discrimination of FSK modulated optical signal using injection-locked DFB lasers
}

\author{
R. Hui \\ S. Benedetto \\ I. Montrosset
}

\begin{abstract}
Direct discrimination of FM and FSK optical signals using injection-locked DFB semiconductor lasers is analysed. The demodulation efficiency is found to be independent of the optical injection level in the steady state. However, the optical injection level is important in determining the dynamic response. There exists an optimum optical injection rate to achieve the highest demodulation speed. The noise analysis revealed that the signal to noise ratio of this method is of the same order as that of the passive interferometer demodulation method.
\end{abstract}

\section{Introduction}

Recently, it was confirmed, both experimentally and theoretically, that the stable locking band of a distributed feedback (DFB) semiconductor laser, in the configuration of external optical injection locking, is symmetrically centred around the frequency of the free-running slave laser (SL) in the low optical injection level. The variation of the junction voltage was measured to be almost linearly related to the frequency detuning throughout the locking band within the unconditionally stable locking regime $[1,2,3]$. This special property of DFB semiconductor lasers permits a simple method to directly demodulate the frequency-modulated (FM) optical signal The first detailed experimental demonstration of this method was recently performed by $H$. Nakajima [3]. The reported results are promising because the method allows the demodulation of optical FM signals directly and without the interferometer.

The purpose of this paper is to present a theoretical analysis of injection-locked DFB semiconductor lasers used as discriminators for FM and FSK optical signals. The static solution of the rate equation reveals that the FM demodulation efficiency is independent of the optical injection level in the steady state. However, the optica injection level is important in determining the dynamic response. Both a small-signal analytical study on FM optical signal demodulation and a large-signal numerical simulation for FSK optical signal demodulation are described. The noise analysis indicates that the signal-tonoise ratio of the present method is in the same order as that of the passive interferometer demodulation method. Some limitations are pointed out and a parameter opti-

Paper 8171J (E7, E13), first received 13th Decernber 1990 and in revised form 25th March 199

The authors are with the Dipartimento Elettronica, Politecnico di Torino, C Duca Abruzzi 24, 10129 Torino, Italy misation is outlined. The limitations imply that the advantages claimed for this method may not be as great as had hitherto been thought [3]

\section{Small-signal analysis}

In DFB semiconductor lasers, the side longitudinalmodes are highly suppressed by the distributed grating, so that the theoretical analysis can be based on the following well known single-mode Van der Pol equation and an equation for the carrier number $[4,5]$ :

$$
\begin{aligned}
d E(t) / d t= & {\left[-i \omega(N)+\left(G(N, I)-1 / \tau_{p}\right) / 2\right] E(t) } \\
& +E_{1}(t) / \tau_{i}+F_{E}(t) \\
d N(t) d t= & C-N(t) / \tau_{s}-G(N, I)|E(t)|^{2}+F_{N}(t)
\end{aligned}
$$

where $E(t)=I^{1 / 2} \exp \{-i[\omega t-\Phi]\}$ is the normalised electric field of the slave laser and $E_{1}(t)=$ $I_{1}^{1 / 2} \exp \left[-i \omega_{1} t\right]$ is the normalised field injected from the master laser (ML). $I$ is the field intensity of SL normalised to the photon number inside the active cavity and $I_{1}$ is the intensity coupled from ML. $N(t)$ is the carrier number, $G(N, I)=G_{N}\left(N-N_{0}\right)+G_{I} I$ is the material gain, $G_{N}$ the differential gain, $G_{I}$ the gain saturation coefficient and $N_{0}$ the carrier number for transparency. $\tau_{p}$ is the photon lifetime, the angular frequency is $\omega(N)=\omega_{s}$ $+(\alpha / 2) G_{N} \Delta N$ where $\Delta N=N-N_{s}$ and $\omega_{s}=\omega\left(N_{s}\right) . \omega_{s}$ and $N_{s}$ are the steady state resonance angular frequency and carrier number of SL, respectively, without optical injection. As the gain nonlinearity is included in this paper, the carrier number is not clamped at its threshold value when the laser operates above threshold. Therefore, we will use the steady state values such as $N_{s}$ and $I_{s}$ for the expansion in the calculation. $\omega_{1}$ and $\omega$ are the angular frequencies of $\mathrm{ML}$ and $\mathrm{SL}, C$ is the carrier injection rate, $\Phi$ is the phase of the injection locked laser field, $\alpha=-2(\delta \omega / \delta N) /(\delta G / \delta N)$ is the linewidth enhancement factor. $\tau_{s}$ is the spontaneous lifetime of $S L$ and $1 / \tau_{i}$ is the coupling constant which can be approximated by $2 n L / c$ with $L$ the cavity length, $n$ the refractive index and $c$ the light speed [5]. $F_{E}(t)$ and $F_{N}(t)$ are the Langevin noise terms.

Recently, it has been revealed that the nonuniform longitudinal intensity distribution, and thus the spatial hole burning effect, may play an important role in determining the linewidth and tunability [6] in DFB semiconductor lasers. In principle, the longitudinal intensity distribution is determined by the $\kappa L$-product, the phase of the grating and other cavity parameters which are different depending on the type of DFB laser. In this paper however, the mean-field approximation is used for simplicity. The major difference between FP and DFB lasers

IEE PROCEEDINGS-J, Vol. 138, No. 4, AUGUST 1991 
considered here is the single longitudinal mode operation of the DFB laser.

In the stable locked state, the frequency of the SL is locked to that of the ML so that the stationary solutions of eqn. 1 are [4]

$$
\begin{aligned}
& \Delta G=-2 \rho \cos \Phi \\
& \Delta \omega=\rho(\sin \Phi-\alpha \cos \Phi)
\end{aligned}
$$

where $\Delta \omega=\omega-\omega_{c}$ is the frequency detuning and $\Delta G=$ $G-1 / \tau_{p} . \rho=\left(I_{1} / I\right)^{1 / 2} / \tau_{i}$ is the normalised optical injection level.

Since $\Delta v=\mu \Delta N$, where $\Delta v$ is the voltage measured at the loading resistor and $\mu$ is a constant related to the laser wavelength and the electric circuits [7], without loss of generality, we only consider the carrier number variation $\Delta N$ in the following for simplicity. The stationary carrier number variation caused by the optical injection is easily obtained as (see Appendix 8)

$$
\Delta N=\frac{-2 \rho \cos \Phi\left[G+G_{I} I\right]}{\left[G G_{N}-G_{I} / \tau_{s}\right]}
$$

From eqns. 2 and 3 , the stationary FM demodulation efficiency can be obtained. The response against frequency detuning is not exactly a straight line, rather, it is the upper portion of an ellipse, similar to the $\Delta G$ against $\Delta \omega$ curve in Reference 4 . The demodulation efficiency in the stable locking range is

$$
\frac{\Delta N}{\Delta f}=\frac{4 \pi \sin \Phi\left(G+G_{I} I\right)}{\left[(\alpha \sin \Phi+\cos \Phi)\left(G G_{N}-G_{I} / \tau_{s}\right)\right]}
$$

where $\Delta f=\Delta \omega / 2 \pi$. It is worth noting that in eqn. 4 , $\Delta N / \Delta f$ is independent of the optical injection ratio. However, as we will show later, this is not true when the dynamic response is considered. Quantitatively, in eqns. 3 and 4, the terms containing $G_{I}$ are negligible compared to the terms with $G$, which indicates that the effect of nonlinear gain saturation is not significant in the steady state. This effect is important, however, in damping the relaxation oscillation in the dynamic aspect as will be analysed later in this paper. On average, the demodulation efficiency of $\Phi=0$ to $\Phi=\pi$ can be approximately evaluated as

$$
\Delta N / \Delta f=4 \pi /\left(\alpha G_{N}\right)
$$

where both $\alpha$ and $G_{N}$ are the commonly used parameters in semiconductor lasers. It should be noticed that this approximation is valid only in the condition of $\alpha \gg 1$. When the value of $\alpha$ is very small, the above mentioned ellipsed will converge toward a circle, so as to make the small signal demodulation efficiency largely dependent on the central frequency of the signal.

The dynamic property of small-signal FM demodulation can be obtained by using a standard treatment as given in Reference 8. This involves linearising eqn. 1 in the small deviations of $\delta I, \delta \Phi$ and $\delta N$ from their equilibrium values and performing the Fourier transformations (see Appendix 8). We get

$$
A(\Omega)=|\delta N(\Omega) / \delta \omega(\Omega)|=|2 \rho I \sin \Phi / D|
$$

with

$$
\begin{aligned}
D= & \left(j \Omega-G_{I} I+R_{S} / I+\rho \cos \Phi\right)(j \Omega+\rho \cos \Phi) \\
& \times\left(j \Omega+G_{N} I+1 / \tau_{s}\right) \\
& +\Omega_{R}^{2}[\rho(\cos \Phi+\alpha \sin \Phi)+j \Phi] \\
& +\rho^{2} \sin ^{2} \Phi\left(j \Omega+G_{N} I+1 / \tau_{s}\right)
\end{aligned}
$$

IEE PROCEEDINGS-J, Vol. 138, No. 4, AUGUST I99I where, $\Omega_{\mathrm{R}}=\left(G G_{N} I\right)^{1 / 2}$ is the relaxation oscillation angular frequency of $\mathrm{SL}, \delta N(\Omega)$ and $\delta \omega(\Omega)$ are the Fourier components of deviations of $N(t)$ and $\omega(t)$, respectively, from their equilibrium values. The small signal demodulation efficiency predicted by eqn. 5 is depicted in Fig. $1 a$ for different optical injection levels at
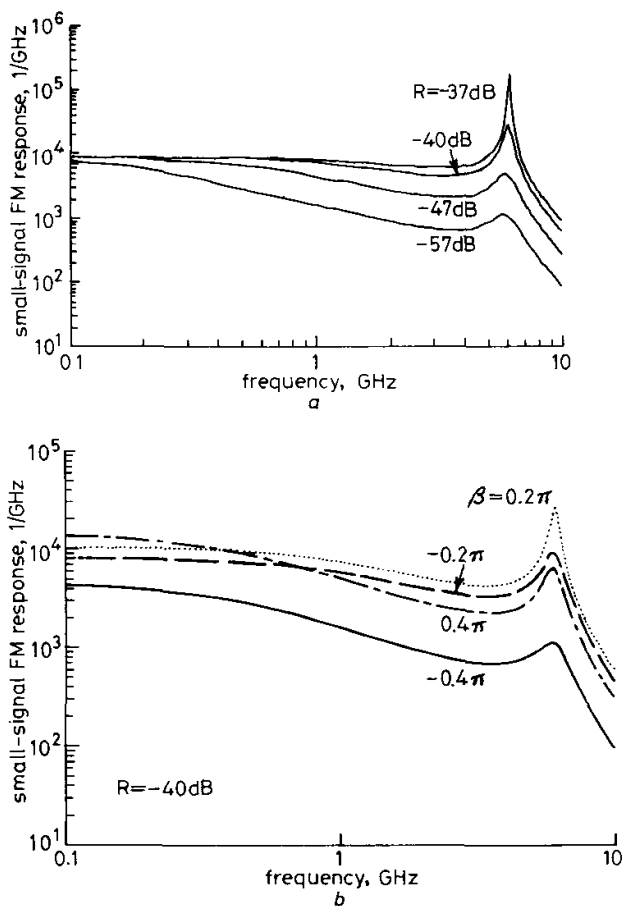

Fig. 1 Small-signal $F M$ demodulation response

a For different optical injection levels $b$ For different relative phase detuning Where $R$ is the optical power injection ratio defined by $R=I_{1} / I$

the centre of the stable locking range around $\beta=0$, where the relative phase detuning is $\beta=\Phi-\tan ^{-1} \alpha$. In Fig. $1 a, R=I_{1} / I$ is defined as the optical injection ratio. Although the demodulation efficiency is independent of the optical injection level in the stationary state as shown in eqn. 4 , discrepancies are found at high modulation frequencies between the curves with different optical injection levels in Fig. 1a. This explains the experimental observation in Reference 3 where this phenomenon was ascribed to the residual intensity modulation. When the optical injection is low, the $3 \mathrm{~dB}$ frequency bandwidth is determined by the half-locking-bandwidth. Alternatively, when the injection is sufficiently high, the relaxation oscillation is more pronounced and sets an upper limit to the optical injection level. This limitation can be approximately evaluated as $1 / \tau_{R}=1 / \tau_{s}+\left(G_{N}-G_{J}\right) I+R_{s} / I$ $+\rho(\cos \Phi-\alpha \sin \Phi)=0$ with $\tau_{R}$ the equivalent damping time, where the relaxation oscillation is undamped $[1,2,11]$. When this dynamic limitation is taken into account, the range of optical signal frequency deviations (dynamic range) of the system $\Delta f_{m}$ is limited to $\Delta f_{m} \leqslant\left[1 / \tau_{s}+R_{s} / I+\left(G_{N}-G_{I}\right) I\right] / 2 \pi$. Because this maximum dynamic range is independent of the linewidth enhancement factor $\alpha$ of the laser, increasing the optical power of the SL is an important way to enlarge the dynamic range without decreasing the demodulation effi- 
ciency. The influence of the relative phase detuning on the small signal FM response is depicted in Fig. $1 b$ for a definite optical injection rate. Obvious asymmetry was
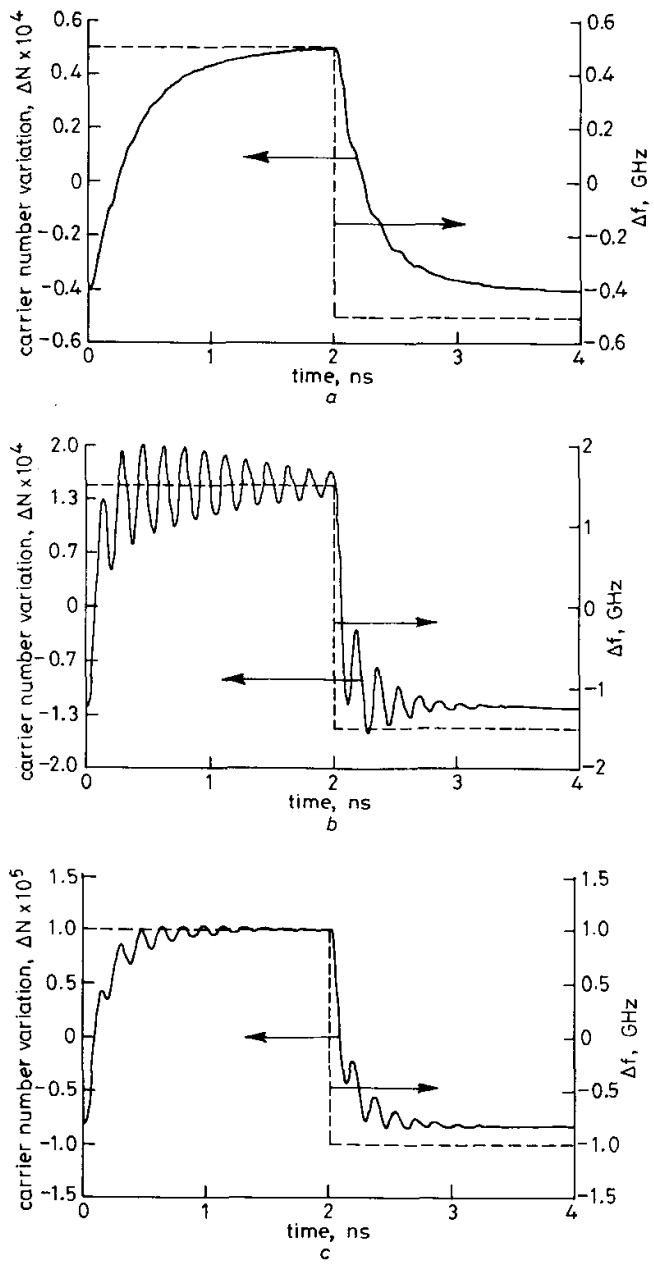

Fig. 2 Results of large-signal simulations for three different optical injection levels

The input optical signal are NRZ bipolar codes with a bit duration of $2 \mathrm{~ns} \Delta N$ is the relative carrier number variation and $\Delta \mathcal{f}$ is the signal frequency deviation $a R=-47 \mathrm{~dB}$

$R=-37 \mathrm{~dB}$
$R=-40.5 \mathrm{~dB}$

found with respect to the zero relative phase detuning. The laser parameters used in this paper are $G_{N}=5.6$ $\times 10^{3} \mathrm{~s}^{-1}, \quad G_{I}=1.8 \times 10^{4} \mathrm{~s}^{-1}, \quad G=6.4 \times 10^{11} \mathrm{~s}^{-1}$, $\Omega_{R}=2 \pi \times 6 \mathrm{GHz}, 1 / \tau_{\mathrm{s}}=3 \times 10^{8} \mathrm{~s}^{-1}, \alpha=6, \tau_{i}=8 \mathrm{ps}$ and $R_{s}=1.28 \times 10^{12}$. With these values, the unconditionally stable locking regime is determined by about $R \leqslant-37.8 \mathrm{~dB}$. It is worthwhile to note that the nonlinear gain saturation effect is the most important effect in determining the equivalent damping time $\tau_{R}$ although it is negligible in the stationary analysis. To the application discussed in this paper, a semiconductor laser with a higher value of $G_{I}$ is preferred. The dynamic range $\Delta f_{m}$ is mainly determined by the value of $G_{I} I$.

\section{3}

Large-signal simulation

In practical digital optical communications, the FSK modulation format is often used. In this case, the injected optical frequency abruptly changes between two values corresponding to ' 0 ' and ' 1 ', and the above small-signal analysis is no longer valid. A large-signal numerical simulation is therefore required. To this end, we integrate the nonlinear, complex field equation (eqn. 1), timedependently using the Fourth-order Runge-Kutta method. The injected frequency signal is switched between the two extremes, which is commonly referred to as the non-return-to-zero (NRZ) bipolar code. Typical results of numerical calculations are shown in Fig. 2.

In the very low optical injection level, the switch-on (and switch-off) time is quite long as shown in Fig. $2 a$. This is because the locking bandwidth is too narrow. In Fig. $2 b$, the optical injection level is too high and the relaxation oscillation is less damped so that the switch time is determined by the time used to damp this relaxation oscillation. Here the obvious difference between the switch-on and the switch-off time can be ascribed to the asymmetric property of $\tau_{R}$ with respect to $\Phi$. Therefore, we can conclude that there exists an optimum value of optical injection level for a certain bit-rate to achieve the highest switch speed.

Usually, the FM index and consequently, the frequency deviation, is limited by the stable locking range. If the frequency deviation of the input signal exceeds this locking range, the demodulated signal, in carrier density variation, is found to randomly oscillate between the two extremes. The maximum carrier number variation attainable is determined by the optical injection level. Nevertheless, in FSK digital transmission systems the minimum value of frequency deviation is related to the bit-rate $B$ as $\Delta F \geqslant 2 B$ with $\Delta F$, the signal frequency difference between ' 0 ' and ' 1 '. The maximum bit-rate attainable is thus limited by the locking bandwidth near the unconditionally stable upper limit [1] i.e. $\Delta F \leqslant \Delta f_{m}$. With the laser parameters listed above and the optical injection level optimised, a switch on (off) time of less than $1 \mathrm{~ns}$ can be obtained as shown in Fig. 2c. This is obtained by carefully adjusting the optical injection level and trading off the two drawback effects mentioned above (slow switching time at low optical injection and relaxation oscillation at higher optical injection). This speed is comparable to another application in which an injection-locked DFB laser was used to achieve optical PSK modulation [9].

\section{Noise analysis}

Unlike the usual passive interferometric FM demodulation method, the injection locked DFB semiconductor lasers, used as the FM demodulation elements, exhibit some special noise properties. Our analysis, so far, was limited to the ideal case, i.e. without noise. In fact, optical signal intensity fluctuations will induce carrier density fluctuations and also variations of the demodulation efficiency in the higher frequency region. Moreover, the Langevin noise will be another source degrading the signal-to-noise ratio.

As mentioned in the small-signal analysis, although the demodulation efficiency is independent of the injected optical intensity in the stationary state, it is intensitydependent at higher modulation frequencies as shown in Fig. 1. The carrier number fluctuation directly caused by the signal intensity fluctuation can be simply evaluated

IEE PROCEEDINGS-J, Vol. 138, No. 4, AUGUST 199 
by using eqn. 3 as

$$
\frac{d N}{d I_{1}}=\frac{-\rho \cos \Phi\left[G+G_{I} I\right]}{I_{1}\left[G G_{N}-G_{I} / \tau_{s}\right]}
$$

The demodulation efficiency variation caused by the signal intensity fluctuation can be obtained through eqn. 5 ,

$$
d A(\Omega) / d I_{1}=[1-(\delta D / \delta \rho) \rho / D] A(\Omega)
$$

The sum of the above two effects could be used to evaluate the influence of the signal intensity fluctuation. However, for simplicity, in the following signal-to-noise ratio analysis only the Langevin noise source will be taken into account.

The contribution of the Langevin noise to the degradation of the receiver signal-to-noise $(S / N)$ ratio can be calculated through the rate equation (eqn. 1). Using the method given in Reference 10 and including the nonlinear gain saturation effect, the power spectrum of the carrier density noise can be obtained as (see Appendix 8)

$$
\begin{aligned}
S_{\mathrm{N}}(\Omega)= & R_{s}\left\{\left|A_{22} A_{31}\right|^{2}(2 I+1)+\left|A_{12} A_{31}\right|^{2} / 2 I\right. \\
& +\left|A_{11} A_{22}-A_{12} A_{12} A_{21}\right|^{2} \\
& +2 R_{e}\left[A _ { 2 2 } A _ { 3 1 } \left(A_{11} A_{22}\right.\right. \\
& \left.\left.\left.-A_{12} A_{21}\right)^{*}\right]\right\} /|Y|^{2}
\end{aligned}
$$

where

$$
\begin{aligned}
A_{11}= & \left(j \Omega-G_{I} I+R_{s} / I+\rho \cos \Phi\right) \\
A_{12}= & 2 I \rho \sin \Phi \\
A_{13}= & -G_{N} I \\
A_{21}= & -\rho \sin \Phi / 2 I \\
A_{22}= & j \Omega+\rho \cos \Phi \\
A_{23}= & \alpha G_{N} / 2 \\
A_{31}= & G \\
A_{33}= & j \Omega+G_{N} I+1 / \tau_{s} \\
Y= & A_{11} A_{22} A_{33}-A_{12}\left(A_{21} A_{33}+A_{23} A_{31}\right) \\
& -A_{13} A_{31} A_{22}
\end{aligned}
$$

In eqn. 8 the noise of ML has been neglected for simplicity. This approximation is verified when we consider the low optical injection limitation in this method as emphasised before. With a low optical injection, the noise spectrum of SL is not influenced by the ML very much [10].

The noise power is $P_{N}=\int_{0}^{B} S_{N}(\Omega) d \Omega$ where $B$ is the signal bandwidth. Consider that the flux of signal photons is related to the optical signal power by an additional scaling factor $h \omega$, with $h$ the Plank constant and $\omega$ the optical frequency. In the ideal case, the frequency deviation of the FM modulated signal equals the stable locking bandwidth, the $S / N$ of the analysed demodulation element can then be evaluated as

$$
(S / N)_{s} \approx \frac{\left|B_{L} A(\Omega)\right|^{2} \tau_{i}}{P_{N} h \omega}
$$

where $B_{L}=\rho\left(1+\alpha^{2}\right)^{1 / 2} / \pi$ is the locking bandwidth. In the conventional FM demodulation scheme, i.e. an interferometer followed by a photodetector, the ideal signalto-noise ratio is $(S / N)_{i}=\eta I_{1} /(h \omega B)$ with $\eta$ the photodiode quantum efficiency. The enhancement of the noise introduced by the semiconductor laser demodulation element can be obtained as

$$
\frac{(S / N)_{i}}{(S / N)_{s}}=\frac{\eta I P_{N} \pi^{2} \tau_{i}}{\left(1+\alpha^{2}\right)|A(\Omega)|^{2} B}
$$

To have a simple comparison, we can assume that $B$ is much lower than the relaxation oscillation frequency, so

IEE PROCEEDINGS-J, Vol. 138, No.4, AUGUST 1991 that $A(\Omega) \approx A(0)=4 \pi /\left(\alpha G_{N}\right), \quad P_{N} \approx S_{N}(0) B$. When the weak optical injection approximation is used $S_{N}(0)=$ $2 R_{s} / I G_{N}^{2}$. Also $\eta=1$ can be assumed for the ideal case. Eqn. 8 can be thus simplified as

$$
\frac{(S / N)_{i}}{(S / N)_{s}}=\frac{R_{s} \tau_{i} \alpha^{2}}{8\left(1+\alpha^{2}\right)}
$$

With the parameter values previously used, eqn. 9 gives a noise enhancement factor of approximately $0 \mathrm{~dB}$. So that, using the injection locked DFB semiconductor lasers for FM signal demodulation, the signal-to-noise ratio is of the same order as the passive interferometer demodulation method. Physically, the signal gain provided by the locked SL is compensated by the Langevin noise generated in it.

\section{Conclusion}

The direct discrimination of FM and FSK optical signals using injection-locked DFB semiconductor lasers has been analysed. The demodulation efficiency was found to be independent of the optical injection level only in the steady state, whereas, in the higher frequency regime this dependence is obvious. A narrow locking bandwidth at very low optical injection levels and the relaxation oscillation at relatively higher injection levels are the two important factors limiting the dynamic response of the demodulation. Both a small-signal analytical study and a large-signal numerical simulation show that there exists an optimum optical injection level to achieve the highest speed of response. Since the spontaneous emission does not contribute significantly to a laser operating well above threshold, the noise enhancement due to the injection-locked laser demodulation element is expected to be less than the laser amplifier demodulator $[5,12]$. The signal-to-noise ratio of the present method is found to be in the same order as that of the passive interferometer demodulation method.

\section{Acknowledgments}

The authors wish to specially thank P. Spano, A. Sapia and A. Mecozzi for helpful discussions. P. Lambkin is also acknowledged for kindly checking the manuscript.

\section{References}

1 HUI, R., MECOZZI, A., D'OTTAVI, A., and SPANO, P.: 'Novel measurement technique of $\alpha$ factor in DFB semiconductor lasers by injection locking', Electron. Lett., 1990, 26, (14), pp. 997-998

2 HUI, R., MECOZZI, A, D'OTTAVI, A., and SPANO, P.: 'Injection locking properties of DFB semiconductor lasers'. IEEE 12th Semiconductor Laser Conf., September 1990, Davos, Switzerland

3 NAKAJIMA, H.: 'Demodulation of multi-gigahertz frequencymodulated optical signal in an injection-locked distributed feedback laser oscillator', Electron. Lett., 1990, 26, (15), pp. 1129-1131

4 HENRY, C.H., OLSSON, N.A., and DUTTA, N.K.: 'Locking range and stability of injection locked $1.54 \mu \mathrm{m}$ InGaAsP semiconductor lasers', IEEE J. Quantum Electron., 1985, QE-21, (8), pp. 1152-1156

5 CHOA, F.S., and KOCH, T.L.: 'Static and dynamical characteristics of narrow-band tunable resonant amplifiers as active filters and of narrow-band tunable resonant amplifiers as active filters and

6 TROMBORG, B., OLESEN, H., and PAN, X.: 'Theory of linewidth for multi-electrode laser diodes with spatially distributed noise sources', (and references therein). IEEE J. Quantum Electron., 1991, QE-27, (2), pp. 178-192

7 MARCUS, D.: 'Heterodyne detection with an injection laser - Part I: principle of operation and conversion efficiency', IEEE $J$. Quantum Electron., 1990, OE-26, (1), pp. 85-93

8·PIAZZOLLA, S., SPANO, P., and TAMBURRINI, M.: 'Smal signal analysis of frequency chirping in injection-locked semicon ductor lasers', IEEE J. Quantum Electron., 1986, QE-22, (12), pp 2219-2223 
9 HUI, R.: 'Optical PSK modulation using injection locked DFB semiconductor lasers', IEEE Photonics Technol. Lett., 1990, 2, (10), pp. 743-746

10 SPANO, P., PIAZZOLLA, S., and TAMBURRINI, M.: 'Frequency and intensity noise in injection-locked semiconductor lasers: Theory and experiments', IEEE J. Quantum Electron., 1986, QE-22, (3), pp. $427-435$

11 LIDOYNE, O., GALLION, P., CHABRAN, C., and DEBARGF G.: 'Locking range, phase noise and power spectrum of an injection locked semiconductor laser', IEE Proc. J., 1990, 137, pp. 147-153

12 KOCH, T.L., CHOA, F.S., HEISMANN, F., and KOREN, U. 'Tunable multiple-quantum-well distributed-Bragg-reflector lasers a tunable narrowband receivers', Electron. Lett., 1989, 25, (14), pp. 890-892

13 HENRY, C.H.: 'Phase noise in semiconductor lasers', IEEE $J$. Lightwave Technol., 1986, LT-4, (3), pp. 298-311

\section{Appendix}

8.1 Derivation of eqn. 3

When the nonlinear gain saturation is considered, the material gain $G(N, I)$ can be linearised around its stationary value without optical injection:

$G(N, I)=G\left(N_{s}, I_{s}\right)+\Delta G$

\section{where}

$$
G\left(N_{s}, I_{s}\right)=1 / \tau_{p}
$$

and

$$
\Delta G=G_{N} \Delta N+G_{I} \Delta I
$$

with

$$
\Delta N=N-N_{s} \text { and } \Delta I=I-I_{s}
$$

In the steady state with optical injection, the carrier number rate equation (eqn. $1 b$ ) reads

$$
\begin{aligned}
{\left[C-N_{s} / \tau_{s}-G I_{s}\right]-\Delta N\left(1 / \tau_{s}\right.} & \left.+G_{N} I_{s}\right) \\
& -\Delta I\left(G_{I} I_{s}+G\right)=0
\end{aligned}
$$

Since the first term in eqn. 4 , representing the operating condition of the free-running slave laser, is zero, we have

$$
\Delta I=\Delta N\left(1 / \tau_{s}+G_{N} I_{s}\right) /\left(G_{I} I_{s}+G\right)
$$

Using eqns. 14,12 and $2 a$, one can directly obtain eqn. 3 .

\subsection{Derivation of eqns. 5 and 8}

Eqn. $1 a$ can be separated into two equations, according to its real and imaginary parts, and then linearised together with eqn. $1 b$ in terms of small deviations around the equilibrium values, $I(t)=I+\delta I(t), \Phi(t)=\Phi+\delta \Phi(t)$,
$N(t)=N+\delta N(t)$, the following linearised equations can be easily obtained:

$$
\begin{aligned}
d \delta I(t) / d t= & \left(G_{I} I-R_{s} I I-\rho \cos \phi\right) \\
& \times \delta I(t)-2 \rho I \sin \Phi \delta \Phi(t) \\
& +G_{N} I \delta N(t)+F_{I}(t) \\
d \delta \Phi(t) / d t= & (\rho \sin \Phi / 2 I) \delta I(t)-\rho \cos \Phi \delta \Phi(t) \\
& -\left(\alpha G_{N} / 2\right) \delta N(t)+F_{\phi}(t) \\
d \delta N(t) / d t= & -G \delta I(t)-\left(1 / \tau_{s}+G_{N} I\right) \delta \Phi(t)+F_{N}(t)
\end{aligned}
$$

where $R_{s}$ is the spontaneous emission rate [13]

The Fourier transformation is used to solve the linear system of equations (eqn. 15) by using the following definition:

$$
y(\Omega)=(2 \pi)^{-1} \int_{-\infty}^{\infty} y(t) \exp (-i \Omega t) d t
$$

Eqn. 15 then becomes

$$
\begin{aligned}
& A_{11} \delta I(\Omega)+A_{12} \delta \Phi(\Omega)+A_{13} \delta N(\Omega)=F_{I}(\Omega) \\
& A_{21} \delta I(\Omega)+A_{22} \delta \Phi(\Omega)+A_{23} \delta N(\Omega)=F_{\phi}(\Omega) \\
& A_{31} \delta I(\Omega)+A_{32} \delta \Phi(\Omega)+A_{33} \delta N(\Omega)=F_{N}(\Omega)
\end{aligned}
$$

where

$$
\begin{aligned}
& A_{11}=\left(j \Omega-G_{I} I+R_{s} / I+\rho \cos \Phi\right) \\
& A_{12}=2 I \rho \sin \Phi \\
& A_{13}=-G_{N} I \\
& A_{21}=-\rho \sin \Phi / 2 I \\
& A_{22}=j \Omega+\rho \cos \Phi \\
& A_{23}=\alpha G_{N} / 2 \\
& A_{31}=G \\
& A_{32}=0 \\
& A_{33}=j \Omega+G_{N} I+1 / \tau_{s}
\end{aligned}
$$

The FM demodulation efficiency is determined by $A(\Omega)=|\delta N(\Omega) / \delta \omega(\Omega)|$, whereas the frequency deviation $\delta \omega(\Omega)$ is related to the phase deviation $\delta \Phi(\Omega)$ by $\delta \omega(\Omega)=j \Omega \delta \Phi(\Omega)$. In this way, we can get eqn. 5 to eqn. 16 by setting all the Langevin noise terms to be zero.

Eqn. 16 is similar to eqn. A3 of Reference 10 except for the inclusion of the nonlinear gain saturation term in eqn. 16 and neglecting the noise of ML for simplicity. Therefore, eqn. 8 can be obtained in the same way as that in Reference 10. 